\title{
LUSTERNIK-SCHNIRELMANN CATEGORY FOR CELL COMPLEXES AND POSETS
}

\author{
KOHEI TANAKA
}

\begin{abstract}
This paper introduces two analogues of the Lusternik-Schnirelmann category from a combinatorial viewpoint. One analogue is defined for finite cell complexes using their subcomplexes and simple homotopy theory; the other is an invariant for finite posets with respect to simple equivalence based on the notion of weak beat points. We examine the relation between these two invariants by taking the face posets of complexes or order complexes of posets.
\end{abstract}

\section{Introduction}

The Lusternik-Schnirelmann category (LS-category for short) of a space $X$ is a fundamental topological (homotopy) invariant, originally introduced by Lusternik and Schnirelmann in [10]. This is simply defined by the minimal number of contractible open subspaces covering $X$.

When a space is equipped with a combinatorial structure (for example, a simplicial complex or cell complex), we can consider some discrete versions of the LS-category. The discrete LS-category of a finite simplicial complex was defined using collapsible subcomplexes instead of contractible open subspaces [1], whereas the simplicial LS-category was introduced as an invariant with respect to strong homotopy [7].

In this paper, we propose another notion of the combinatorial (geometric) $L S$-category $\operatorname{cgcat}(Y)$ for a (regular) finite cell complex $Y$ using contractible subcomplexes. To make this a simple homotopy invariant, we introduce the combinatorial strong LS-category cCat $(Y)$ based on the strong LS-category concept introduced by Ganea [9]. Throughout the paper, $X$ will denote

Received December 10, 2015; received in final form February 25, 2016.

This work was supported by JSPS KAKENHI Grant Number 15K17535.

2010 Mathematics Subject Classification. Primary 55M30. Secondary 57Q10. 
a general topological space, whereas, for distinction, $Y$ will be a (regular) cell complex.

There is another notion of a modified LS-category using weakly contractible open subspaces. This is called the quasi-(geometric) LS-category qgcat $(X)$ of a space $X$. Using Ganea's idea again, the quasi-strong LS-category qCat $(X)$ can be naturally defined as a weak homotopy (simply equivalent) invariant. When $X$ is a finite space, these are closely related to the combinatorial LScategory defined above. A finite space can be regarded as a finite poset [2], [12]. Hence, we will identify both.

THEOREM 3.15. Let $Y$ be a finite regular cell complex. The following equality holds:

$$
\operatorname{cgcat}(Y)=\operatorname{qgcat}(\chi(Y)) \text {, }
$$

where $\chi(Y)$ is the face poset of $Y$.

The order complex $\mathcal{K}(P)$ of a poset $P$ is (the geometric realization of ) a simplicial complex consisting of ordered sequences of $P$ formed of $p_{0}<\cdots<p_{n}$. This is known as an inverse operation for taking face posets, because $\mathcal{K}(\chi(Y))$ and $Y$ are isomorphic for any regular cell complex $Y$. We naturally expect that an equality similar to the above will hold: $\operatorname{cgcat}(\mathcal{K}(P))=\operatorname{qgcat}(P)$ for a finite poset $P$. In general, however, this is not true. The equality holds in the case of the strong version of each LS-category.

TheOREM 3.17. Let $P$ be a finite poset. The following equality holds:

$$
\operatorname{cCat}(\mathcal{K}(P))=\mathrm{qCat}(P) \text {. }
$$

The classical LS-category was originally defined as a lower bound for the number of critical points of a Morse function on some manifold $M$. A discrete version of this property for a discrete Morse function [8] on a simplicial complex and discrete LS-category was derived in [1]. We present a simple proof of this property for general finite regular cell complexes and our combinatorial LS-category.

THEOREM 4.4. Given a discrete Morse function on a finite regular cell complex $Y$ with $n+1$ critical cells, we have the following inequality:

$$
\operatorname{cgcat}(Y) \leq n
$$

The remainder of this paper is organized as follows. Section 2 recalls some basic notions with respect to the classical LS-category. We introduce the notion of a combinatorial LS-category based on subcomplexes and simple homotopy theory. Section 3 yields another invariant of spaces, the quasi-LScategory based on weak homotopy equivalence. We focus on the case of finite posets, and compare the combinatorial LS-category. In Section 4, we prove a discrete analogue of the Lusternik-Schnirelmann theorem for a discrete Morse function on a finite regular cell complex. 


\section{LS-category for regular cell complexes and simple homotopy theory}

We first introduce the definition of the geometric LS-category of a space. This is a topological invariant, but is not a homotopy invariant of spaces [6].

Definition 2.1. Let $X$ be a space. The geometric LS-category gcat $(X)$ is the smallest non-negative integer $n$ such that $X$ can be covered by $n+1$ open subspaces that are contractible in themselves.

On the other hand, the LS-category is a homotopy invariant defined using open subspaces that are contractible in $X$, but not in themselves.

Definition 2.2. The $L S$-category $\operatorname{cat}(X)$ of a space $X$ is the smallest nonnegative integer $n$ such that $X$ can be covered by $n+1$ open subspaces that are contractible in $X$.

The following describes another way to make the geometric LS-category a homotopy invariant.

Definition 2.3. Let $X$ be a space. The strong LS-category $\operatorname{Cat}(X)$ is the minimum value of the geometric LS-category cat $\left(X^{\prime}\right)$ over all spaces $X^{\prime}$ having the homotopy type of $X$ :

$$
\operatorname{Cat}(X):=\min \left\{\operatorname{gcat}\left(X^{\prime}\right) \mid X^{\prime} \simeq X\right\} .
$$

The above definitions immediately give the inequalities cat $(X) \leq \operatorname{gcat}(X)$ and $\operatorname{Cat}(X) \leq \operatorname{gcat}(X)$ for any space $X$. Takens [13] derived the following relation between the LS-category and the strong LS-category of a space $X$ :

$$
\operatorname{cat}(X) \leq \operatorname{Cat}(X) \leq \operatorname{cat}(X)+1 .
$$

When $X$ is a CW-complex, we can consider an analogue of the LS-category using its subcomplexes.

Definition 2.4. Let $Y$ be a $\mathrm{CW}$-complex. The combinatorial LS-category $\operatorname{cgcat}(Y)$ is the smallest non-negative integer $n$ such that $Y$ can be covered by $n+1$ subcomplexes that are contractible in themselves.

The combinatorial LS-category definition above was introduced in [1] (it was called the closed geometric category). A subcomplex $A$ of a $\mathrm{CW}$-complex admits an open neighborhood $U$ with a deformation retraction onto $A$. This property guarantees the following inequality.

Proposition 2.5 (Theorem 7 of [1]). The following inequality holds for a $C W$-complex $Y$ :

$$
\operatorname{gcat}(Y) \leq \operatorname{cgcat}(Y) \text {. }
$$

With respect to the combinatorial LS-category, the following subadditivity property holds, because contractible subcomplexes in a subcomplex of a CWcomplex remain in the total complex. 
Proposition 2.6. Let a $C W$-complex $Y$ be the union of subcomplexes $A$ and $B$ of $Y$. The following subadditivity with respect to the combinatorial LS-category holds:

$$
\operatorname{cgcat}(Y) \leq \operatorname{cgcat}(A)+\operatorname{cgcat}(B)+1 .
$$

A cell complex (CW-complex) is called regular if every characteristic map is a homeomorphism onto its image. For finite regular cell complexes, some combinatorial versions of homotopy theory have been considered in the literature. One of these is known as simple homotopy theory [14].

Definition 2.7. A face $d$ of a cell $e$ in a regular cell complex is called free when $e$ is a unique coface of $d$ with $\operatorname{dim} e=\operatorname{dim} d+1$.

Let $Y$ and $Y^{\prime}$ be two finite regular cell complexes. We say that $Y$ collapses to $Y^{\prime}$ by an elementary collapse, or that $Y^{\prime}$ extends to $Y$ by an elementary expansion, if $Y^{\prime}$ is the subcomplex formed by removing a cell together with a free face of $Y$. We denote this by $Y$ e $\nearrow Y^{\prime}$, or $Y \searrow Y^{\prime}$.

Two finite regular cell complexes $Y$ and $Y^{\prime}$ are simple homotopy equivalent to each other if there exists a sequence $Y=Z_{1}, Z_{2}, \ldots, Z_{n}=Y^{\prime}$ of finite regular cell complexes such that $Z_{i} \searrow Z_{i+1}$ or $Z_{i}$ e $Z_{i+1}$ for all $i$. We denote this by $Y \wedge Y^{\prime}$. In particular, when $Y^{\prime}$ is a single point and $Z_{i} \searrow Z_{i+1}$ for all $i$, we say that $Y$ is elementary collapsible.

If a finite regular cell complex $Y$ is simple homotopy equivalent to another complex $Y^{\prime}$, then $Y$ is clearly homotopy equivalent to $Y^{\prime}$. However, the converse is not generally true. This relation is controlled by Whitehead torsion or the Whitehead group of the fundamental group (see [14] for details). It depends only on the fundamental group; hence, two simply connected spaces are homotopy equivalent if and only if they are simple homotopy equivalent.

The combinatorial geometric LS-category is not homotopy invariant, like the standard geometric LS-category. As the following example demonstrates, it is not even simple homotopy invariant.

ExAmPle 2.8. Consider the following two cell decompositions of the torus $T^{2}=S^{1} \times S^{1}$. We will show that $\operatorname{cgcat}\left(T^{2}\right)=3$ while $\operatorname{cgcat}\left(\operatorname{sd}\left(T^{2}\right)\right)=2$.

As a classical result, it is well known that $\operatorname{gcat}\left(T^{2}\right)=2$. Proposition 2.5 guarantees the inequality $\operatorname{cgcat}\left(T^{2}\right) \geq 2$ for any cell decomposition on $T^{2}$.

(1) A circle $S^{1}$ possesses the minimal regular cell decomposition with two 0-cells and two 1-cells:

$$
S^{1}=e_{+}^{0} \cup e_{-}^{0} \cup e_{+}^{1} \cup e_{-}^{1} .
$$

This gives rise to the product cell decomposition on $T^{2}$ shown on the lefthand side of Figure 1. This can be covered by four closed 2-cells; hence, we have $\operatorname{cgcat}\left(T^{2}\right) \leq 3$. However, $\operatorname{cgcat}\left(T^{2}\right) \neq 2$ for this cell decomposition. If $\operatorname{cgcat}\left(T^{2}\right)=2$, there exist three contractible subcomplexes $A_{1}, A_{2}$, and $A_{3}$ covering $T^{2}$. Thus, some $A_{i}$ must include at least two closed 2-cells. 

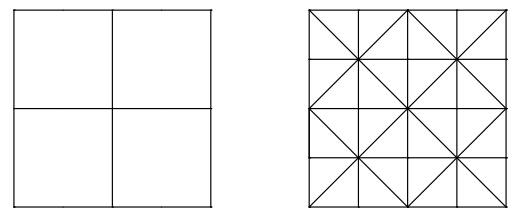

Figure 1. Two cell decompositions of the torus (the upper boundary is identified with the lower one, and the right-hand boundary is identified with the left-hand one for each square).
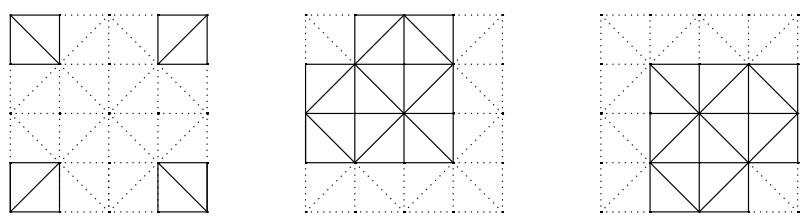

FiguRE 2. Three contractible subcomplexes in $\operatorname{sd}\left(T^{2}\right)$.

This is not contractible, which leads to a contradiction. It turns out that $\operatorname{cgcat}\left(T^{2}\right)=3$.

(2) Next, we consider the barycentric subdivision $\operatorname{sd}\left(T^{2}\right)$ of $T^{2}$ with the decomposition shown on the right-hand side of Figure 1. Unlike the previous case, we are able to choose three contractible subcomplexes because of the finer decomposition (Figure 2). It turns out that $\operatorname{cgcat}\left(\operatorname{sd}\left(T^{2}\right)\right)=2$.

The barycentric subdivision $\operatorname{sd}(Y)$ of a regular cell complex $Y$ is simple homotopy equivalent to the original complex $Y$ (see Theorem 3.9). The examples above show that the combinatorial LS-category is not simple homotopy invariant.

As the example above shows, the following inequality holds in general, because a subcomplex of $Y$ is also a subcomplex of the barycentric subdivision $\operatorname{sd}(Y)$.

Proposition 2.9. For a finite regular cell complex $Y$, the following inequality holds:

$$
\operatorname{cgcat}(\operatorname{sd}(Y)) \leq \operatorname{cgcat}(Y) \text {. }
$$

To make the combinatorial LS-category simple homotopy invariant, we naturally conceive an analogue of the strong LS-category in Definition 2.3.

Definition 2.10. Let $Y$ be a finite regular cell complex. The combinatorial strong $L S$-category cCat $(Y)$ is the minimum value of the combinatorial LScategory $\operatorname{cgcat}\left(Y^{\prime}\right)$ over all finite regular cell complexes $Y^{\prime}$ having the simple 
homotopy type of $Y$ :

$$
\operatorname{cCat}(Y):=\min \left\{\operatorname{cgcat}\left(Y^{\prime}\right) \mid Y^{\prime} \wedge Y\right\}
$$

\section{LS-category for finite posets and weak beat points}

This section proposes another analogue of the LS-category. The idea of the definition is to use weakly contractible covers instead of contractible covers.

Definition 3.1. Let $X$ be a space. The quasi-geometric LS-category qgcat $(X)$ is the smallest nonnegative integer $n$ such that $X$ can be covered by $n+1$ weakly contractible (homotopically trivial) open subspaces.

This definition immediately gives the inequality $\operatorname{qgcat}(X) \leq \operatorname{gcat}(X)$ for any space $X$. We can state the following subadditivity property with respect to the quasi-geometric LS-category in a similar way to Proposition 2.6.

Proposition 3.2. Let a space $X$ be the union of open subspaces $A$ and $B$ of $X$. The following subadditivity with respect to the quasi-geometric $L S$ category holds:

$$
\operatorname{qgcat}(X) \leq \operatorname{qgcat}(A)+\operatorname{qgcat}(B)+1
$$

We are interested in the case when $X$ is a finite poset (space). A poset $P$ can be regarded as a $T_{0}$-Alexandroff space whose underlying set is $P$ with the topology generated from all ideals of $P$. Here, an ideal of a poset $P$ is a subposet $Q$ that is closed under the lower order, that is, any element $p$ in $P$ belongs to $Q$ if $p \leq q$ for some $q$ in $Q$. Conversely, for any $T_{0}$-Alexandroff space $X$, the underlying set is equipped with a partial order $x \leq y$ defined by $x \in \bar{y}$, where $\bar{y}$ is the minimal open set including $y$. These correspondences ensure that the category of posets is equivalent to the category of $T_{0}$-Alexandroff spaces. For this reason, we regard a poset as a $T_{0}$-Alexandroff space throughout this paper. In particular, a finite poset can be regarded as a finite $T_{0}$-space.

The notions of beat points and weak beat points play an important role in homotopy theory for posets.

Definition 3.3. Let $P$ be a poset. An element $x$ in $P$ is called an up-beat point if the subposet $\{y \in P \mid x>y\}$ possesses a unique maximal element. Down-beat points are defined dually. We refer to a point as simply a beat point if it is either an up-beat point or a down-beat point.

A beat point of a poset does not affect the homotopy type. We obtain a minimal model with respect to the homotopy type of a finite poset by removing all beat points one by one. This is called the core of a poset. It is well known that the core is determined uniquely up to isomorphism, regardless of the order in which the points are removed. Stong classified the homotopy type of finite posets using their cores. 
Theorem 3.4 (Theorem 4 of [12]). Let $P$ and $Q$ be finite posets. $P$ is homotopy equivalent to $Q$ if and only if their cores are isomorphic to each other. In particular, a finite poset $P$ is contractible if and only if the core consists of a single point.

Weak beat points are a generalization of beat points.

Definition 3.5. Let $P$ be a poset. A point $x$ in $P$ is called a weak up-beat point if the subposet $\{y \in P \mid x>y\}$ is contractible. Weak down-beat points are defined dually. We refer to a point as simply a weak beat point if it is either a weak up-beat point or a weak down-beat point.

A weak beat point of a poset does not affect the weak homotopy type or the homotopy type of the order complex. We can consider an analogue of the core as a minimal finite model formed by removing all weak beat points. Note that the core is determined uniquely up to isomorphism, but the minimal finite model is not [3], [4].

The following Wallet example clarifies the notions of beat points and weak beat points.

Example 3.6 (Example 4.2.1 of [2]). Consider the poset described as the following Hasse diagram.

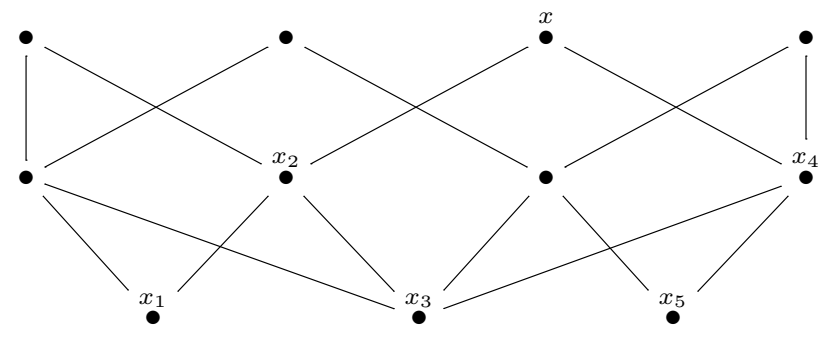

It does not have beat points; however, the point $x$ is a weak beat point. Indeed, the subposet $\{y \in P \mid x>y\}$ can be written as $x_{1}<x_{2}>x_{3}<x_{4}>x_{5}$, and is contractible. By removing the point $x$ from the diagram, the subposet does have beat points and is contractible. Consequently, the original poset is not contractible, but weakly contractible (the order complex is contractible).

Definition 3.7. Let $P$ and $Q$ be two finite posets. We say that $P$ collapses to $Q$ by an elementary collapse, or $Q$ expands to $P$ by an elementary expansion, if $Q$ is the subposet of $P$ formed by removing a weak beat point. We denote this by $P \searrow Q$, or $Q$ e $P$.

Two finite posets $P$ and $Q$ are simply equivalent to each other if there exists a sequence $P=P_{1}, P_{2}, \ldots, P_{n}=Q$ of finite posets such that $P_{i} \searrow_{\searrow}^{e} P_{i+1}$ or $P_{i}$ e` $P_{i+1}$ for all $i$. We denote this by $P \wedge Q$. 
This definition of simple equivalence for finite posets is similar to that of simple homotopy equivalence for complexes in Definition 2.7. These correspond to one another by taking order complexes, or face posets.

Definition 3.8. Let $P$ be a poset and $Y$ be a cell complex.

(1) The face poset $\chi(Y)$ consists of cells of $Y$ with the order relation given by $d \leq e$ if $d \subset \bar{e}$.

(2) The order complex $\mathcal{K}(P)$ is a simplicial complex whose $n$-simplexes are ordered sequences $p_{0}<\cdots<p_{n}$ in $P$.

For a cell complex $Y$, the barycentric subdivision $\operatorname{sd}(Y)$ is (the geometric realization of) the order complex of the face poset of $Y$. Similarly, for a poset $P$, the barycentric subdivision $\operatorname{sd}(P)$ (we use the same symbol as for complexes) is the face poset of the order complex of $P$.

The following are fundamental properties of order complexes and face posets. For more details, we refer readers to [11], [5], and [4].

TheOREM 3.9. Let $P$ be a finite poset and $Y$ be a finite regular cell complex.

(1) A natural map $\mathcal{K}(P) \rightarrow P$ is a weak homotopy equivalence (not generally a homotopy equivalence).

(2) $\operatorname{sd}(P)$ and $P$ are simply equivalent to each other.

(3) $\operatorname{sd}(Y)$ and $Y$ are simple homotopy equivalent to each other.

Theorem 3.10 (Theorem 3.10 of [4]). Let $P, Q$ be finite posets, and $Y, Z$ be finite regular cell complexes.

(1) $P$ is simply equivalent to $Q$ if and only if the order complex $\mathcal{K}(P)$ is simple homotopy equivalent to $\mathcal{K}(Q)$.

(2) $Y$ is simple homotopy equivalent to $Z$ if and only if the face poset $\chi(Y)$ is simply equivalent to $\chi(Z)$.

Barmak and Minian originally proved the second statement for simplicial complexes [4]. It is not difficult to extend this result to regular cell complexes. For a finite regular cell complex $Y$, the barycentric subdivision $\operatorname{sd}(Y)$ is a simplicial complex that is simple homotopy equivalent to the original $Y$. This implies that $Y$ is simple homotopy equivalent to $Z$ if and only if $\operatorname{sd}(Y)$ is $\operatorname{sim}$ ply equivalent to $\operatorname{sd}(Z)$. Similarly, $\chi(\operatorname{sd}(Y))=\operatorname{sd}(\chi(Y))$ is simply equivalent to $\chi(Y)$. This completes the second statement in the above theorem.

The next corollary follows from Theorem 3.10, Whitehead's simple homotopy theorem, and McCord's theorem.

Corollary 3.11. Let $P$ be a finite poset. The following are equivalent:

(1) $P$ is weakly contractible.

(2) The order complex $\mathcal{K}(P)$ is contractible.

(3) $P$ is simply equivalent to a single point. 
Proof. $1 \Leftrightarrow 2$ : McCord's theorem states that the natural map $\mathcal{K}(P) \rightarrow P$ is a weak homotopy equivalence. A poset $P$ is weakly contractible if and only if $\mathcal{K}(P)$ is contractible, since $\mathcal{K}(P)$ is a simplicial complex.

$2 \Leftrightarrow 3$ : For an arbitrary simplicial complex $Y$, being contractible is equivalent to being simple homotopy equivalent to a single point, since the Whitehead group $\mathrm{Wh}\left(\pi_{1}(Y)\right)$ is trivial. Theorem 3.10 completes the result.

Let us again discuss the quasi-geometric LS-category for a finite poset. Recalling the topology of a finite poset $P$ given at the start of Section 3 , the quasi-geometric LS-category qgcat $(P)$ is the smallest nonnegative integer $n$ such that $P$ can be covered by $n+1$ weakly contractible ideals. Unfortunately, the quasi-geometric LS-category is not invariant with respect to weak homotopy equivalence or simple equivalence. For example, consider the face poset $\chi\left(T^{2}\right)$ of the torus with cell decomposition (1) in Example 2.8. Then, we have the strict inequality qgcat $\left(\chi\left(T^{2}\right)\right)>\operatorname{qgcat}\left(\operatorname{sd}\left(\chi\left(T^{2}\right)\right)\right)$, even though $\chi\left(T^{2}\right)$ and the barycentric subdivision $\operatorname{sd}\left(\chi\left(T^{2}\right)\right)$ are simply equivalent (weak homotopy equivalent) to each other (see Remark 3.16 and Theorem 3.15 for more details).

To improve this situation, we propose the next invariant as a discrete analogue of the strong LS-category for finite posets.

Definition 3.12. Let $P$ be a finite poset. The quasi-strong LS-category $\mathrm{qCat}(P)$ is the minimum value of the quasi-geometric LS-category qgcat $(Q)$ over all finite posets $Q$ that are simply equivalent to $P$ :

$$
\mathrm{qCat}(P):=\min \{\operatorname{qgcat}(Q) \mid Q \wedge P\} .
$$

REMARK 3.13. In a more general way, we can define a weak homotopy invariant for an arbitrary space $X$ as the minimum value of the quasi-geometric LS-category qgcat $\left(X^{\prime}\right)$ over all spaces $X^{\prime}$ that are weak homotopy equivalent to $X$. However, weak homotopy equivalent finite posets $X$ and $X^{\prime}$ are not, in general, simply equivalent to each other (the converse is true). This holds when the Whitehead group $\mathrm{Wh}\left(\pi_{1}(X)\right)$ is trivial. As we have seen in Theorem 3.10, the notion of simple equivalence in finite posets is closely related to simple homotopy theory in finite complexes. For this reason, we adopt a definition of the quasi-strong LS-category for finite posets using simple equivalence.

REMARK 3.14. Our concept of a combinatorial analogue of the LS-category using simple homotopy and weak beat points of posets has already been mentioned, although no details were given, in Remark 3, Section 4 of [7].

We now derive the relation between the combinatorial geometric (strong) LS-category and the quasi-geometric (strong) LS-category. 
THEOREM 3.15. Let $Y$ be a finite regular cell complex. We have the following equality:

$$
\text { qgcat }(\chi(Y))=\operatorname{cgcat}(Y) \text {. }
$$

Proof. If $\operatorname{cgcat}(Y)=n$, there exist contractible subcomplexes $A_{0}, \ldots, A_{n}$ covering $Y$. These face posets $\chi\left(A_{0}\right), \ldots, \chi\left(A_{n}\right)$ are ideals, and cover $\chi(Y)$. By Corollary 3.11, each $\chi\left(A_{k}\right)$ is weakly contractible, since $\mathcal{K}\left(\chi\left(A_{k}\right)\right)$ is homotopy equivalent to $A_{k}$ and a single point. Thus, we have the inequality qgcat $(\chi(Y)) \leq \operatorname{cgcat}(Y)$.

Next, we will show the converse inequality. If qgcat $(\chi(Y))=n$, there exist weakly contractible ideals $I_{0}, \ldots, I_{n}$ covering $\chi(Y)$. For each ideal $I_{k}$, any face of a cell in $I_{k}$ again belongs to $I_{k}$. This implies that the union $Y_{k}=$ $\bigcup_{e \in I_{k}} e$ is closed, and this is a subcomplex of $Y$ with the face poset $I_{k}$. These subcomplexes $Y_{0}, \ldots, Y_{n}$ cover $Y$, and each $Y_{k}$ is contractible, since $\operatorname{sd}\left(Y_{k}\right)=$ $\mathcal{K}\left(I_{k}\right)$ is contractible by Corollary 3.11 and Theorem 3.9. This gives the desired inequality qgcat $(\chi(Y)) \geq \operatorname{cgcat}(Y)$.

REMARK 3.16. Note that $\operatorname{cgcat}(\mathcal{K}(P)) \neq$ qgcat $(P)$ for some finite poset $P$. The inequality $\operatorname{cgcat}(\mathcal{K}(P)) \leq \operatorname{qgcat}(P)$ always holds, whereas the converse inequality is not always true. Consider the face poset $\chi\left(T^{2}\right)$ of the torus with cell decomposition (1) in Example 2.8. We have the following strict inequality:

$$
\operatorname{cgcat}\left(\mathcal{K}\left(\chi\left(T^{2}\right)\right)\right)=\operatorname{cgcat}\left(\operatorname{sd}\left(T^{2}\right)\right)=2<3=\operatorname{cgcat}\left(T^{2}\right)=\operatorname{qgcat}\left(\chi\left(T^{2}\right)\right) .
$$

Even though $\operatorname{cgcat}(\mathcal{K}(-)) \neq$ qgcat $(-)$ as above, the equality always holds when passing to the strong version.

TheOREm 3.17. Let $P$ be a finite poset. We have the following equality:

$$
\operatorname{cCat}(\mathcal{K}(P))=\mathrm{qCat}(P) .
$$

Proof. If $\operatorname{cgcat}(P)=n$, there exist weakly contractible ideals $I_{0}, \ldots, I_{n}$ covering $P$. These order complexes are contractible subcomplexes, and cover $\mathcal{K}(P)$. Hence, we have the following inequality:

$$
\operatorname{qgcat}(\operatorname{sd}(P)) \leq \operatorname{cgcat}(\mathcal{K}(P)) \leq \operatorname{qgcat}(P) .
$$

Theorem 3.10 guarantees that this inequality is preserved in the case of the combinatorial or quasi-strong LS-category:

$$
\mathrm{qCat}(\operatorname{sd}(P)) \leq \mathrm{cCat}(\mathcal{K}(P)) \leq \mathrm{qCat}(P) .
$$

Since the barycentric subdivision $\operatorname{sd}(P)$ and the original poset $P$ are simply equivalent to each other, the equality $\mathrm{cCat}(\operatorname{sd}(P))=\mathrm{cCat}(P)$ holds. This leads to the desired result:

$$
\operatorname{cCat}(\mathcal{K}(P))=\mathrm{qCat}(P) .
$$

Theorem 3.15 immediately leads to the following result for face posets. 
Corollary 3.18. Let $Y$ be a finite regular cell complex. We have the following equality:

$$
\mathrm{qCat}(\chi(Y))=\operatorname{cCat}(Y) .
$$

\section{Discrete Morse theory and the Lusternik-Schnirelmann theorem}

The classical Lusternik-Schnirelmann theorem relates the LS-category of a closed manifold $M$ with the number of critical points of a Morse function $f$, i.e., the inequality cat $(M) \leq n$ holds if $f$ has $n+1$ critical points. A discrete analogue of Morse functions was introduced by Forman [8].

Definition 4.1. Let $Y$ be a finite regular cell complex. A discrete Morse function on $Y$ is a real-valued function $f: \chi(Y) \rightarrow \mathbb{R}$ on the face poset of $Y$ satisfying the following two conditions:

(1) For any cell $e \in \chi(Y)$, the number of codimension-one faces $d$ of $e$ that satisfy $f(e) \leq f(d)$ is at most one.

(2) For any cell $d \in \chi(Y)$, the number of cells $e$ having $d$ as a codimensionone face that satisfy $f(d) \geq f(e)$ is at most one.

A discrete Morse function on $Y$ yields an acyclic matching on the face poset $\chi(Y)$, that is, a pair of a cell $e$ and its codimension-one face $d$ is matched if $f(e) \leq f(d)$. Unmatched cells are said to be critical. This separates the face posets into three distinct subsets: $\chi(Y)=D \amalg C \amalg U$, where:

(1) $D$ denotes the set of cells matched with co-boundary cells.

(2) $C$ denotes the set of critical cells.

(3) $U$ denote the set of cells matched with boundary cells.

The discrete Morse function is equipped with a bijection $\mu_{f}: D \rightarrow U$ given by $\mu_{f}(d)=e$ for the matched pair $(d, e)$.

A discrete version of the Lusternik-Schnirelmann theorem was shown in [1] for discrete Morse functions on simplicial complexes. It is not difficult to extend this theorem to regular cell complexes. The following definition of the discrete LS-category is based on Definition 8 in [1].

Definition 4.2. Let $Y$ be a finite regular cell complex. The discrete geometric $L S$-category $\operatorname{dgcat}(Y)$ (denoted by $\widetilde{\operatorname{dcat}}(Y)$ and called the discrete geometric pre-category in [1]) is the smallest non-negative integer such that $Y$ can be covered by $n+1$ elementary collapsible subcomplexes. The discrete strong $L S$-category $\operatorname{dCat}(Y)$ (denoted by $\operatorname{dcat}(Y)$ and called the discrete category in [1]) is the minimum value of the discrete geometric LS-category $\operatorname{dgcat}\left(Y^{\prime}\right)$ over all finite regular cell complexes $Y^{\prime}$ such that $Y$ collapses to $Y^{\prime}$ :

$$
\operatorname{dCat}(Y):=\min \left\{\operatorname{dgcat}\left(Y^{\prime}\right) \mid Y \searrow Y^{\prime}\right\} .
$$


Elementary collapse is a special case of the simple homotopy in Definition 2.7. Thus, it is obvious that $\operatorname{cgcat}(Y) \leq \operatorname{dgcat}(Y)$ and $\operatorname{cCat}(Y) \leq$ $\operatorname{dCat}(Y)$ for every finite regular cell complex $Y$. Furthermore, the above definition implies that $\operatorname{dCat}(Y) \leq \operatorname{dgcat}(Y)$. Theorem 26 in [1] states that $\operatorname{dCat}(Y) \leq n$ for a discrete Morse function on a simplicial complex $Y$ with $n+1$ critical cells.

We will present a simple proof of the above theorem for the case of $\operatorname{cgcat}(Y)$ instead of $\operatorname{dCat}(Y)$ for a general finite regular cell complex $Y$. Our proof uses the subadditivity of the combinatorial geometric LS-category.

Definition 4.3. Given a discrete Morse function $f$ on a regular cell complex $Y$, the level subcomplex $Y(a)$ at $a \in \mathbb{R}$ is a subcomplex of $Y$ generated from cells $e$ with $f(e) \leq a$ :

$$
Y(a)=\bigcup_{f(e) \leq a}\left(\bigcup_{e^{\prime} \subset \bar{e}} e^{\prime}\right) .
$$

Similarly, for $b<a$ in $\mathbb{R}$, we define $Y(b, a)$ to be a subcomplex of $Y$ generated from cells $e$ with $b \leq f(e) \leq a$.

Referring to the proof of Theorem 3.3 in [8], we may assume that our discrete Morse function $f$ is injective. That is, we can perturb $f$ to an injective discrete Morse function without affecting which cells are critical.

THEOREM 4.4. Given an injective discrete Morse function $f$ on $Y$ with $n+1$ critical cells, we have the following inequality:

$$
\operatorname{cgcat}(Y) \leq n
$$

Proof. By the assumption of the injectivity of $f$, the critical cells can be ordered as $c_{0}, \ldots, c_{n}$ such that $f\left(c_{i}\right)<f\left(c_{i+1}\right)$ for any $i$. Furthermore, we can choose a real number $a_{i}$ as $f\left(c_{i}\right)-\varepsilon$ for a sufficiently small $\varepsilon>0$ such that there is no cell $e$ with $a_{i} \leq f(e)<f\left(c_{i}\right)$. For convenience, define $a_{n+1}$ as the maximal value of $f$. It suffices to show that $\operatorname{cgcat}\left(Y\left(a_{i}\right)\right) \leq i-1$ for each $i$. We proceed by induction.

When $i=1$, the level subcomplex $Y\left(a_{1}\right)$ contains only one critical cell $c_{0}$. This is a 0-cell, and takes the minimal value of $f$ (see Example 2.3 of [8]). Since cell $d$ in $Y\left(a_{1}\right)$ with the maximal value of $f$ is a free face, we can collapse $Y\left(a_{1}\right)$ onto $Y\left(a_{1}\right) \backslash\left(d \cup \mu_{f}(d)\right)$. By repeating this process, $Y\left(a_{1}\right)$ can be collapsed onto the critical 0 -cell $c_{0}$, and $\operatorname{cgcat}\left(Y\left(a_{1}\right)\right)=0$.

Assume that $\operatorname{cgcat}\left(Y\left(a_{i}\right)\right) \leq i-1$. The level subcomplex $Y\left(a_{i+1}\right)$ is the union of $Y\left(a_{i}\right)$ and $Y\left(a_{i}, a_{i+1}\right)$. Here, the subcomplex $Y\left(a_{i}, a_{i+1}\right)$ can be collapsed onto $Y\left(a_{i}, f\left(c_{i}\right)\right)$ by repeatedly collapsing the pair of cells $d$ and $\mu_{f}(d)$, where $d$ is the cell of $Y\left(a_{i}, a_{i+1}\right)$ with the maximal value of $f$. The subcomplex $Y\left(a_{i}, f\left(c_{i}\right)\right)$ simply forms the closed cell $\overline{c_{i}}$. This is homotopy equivalent to a single point; hence, $\operatorname{cgcat}\left(Y\left(a_{i}, a_{i+1}\right)\right)=0$. The subadditivity 
(Proposition 2.6) with respect to the combinatorial geometric LS-category gives the desired inequality:

$$
\begin{aligned}
\operatorname{cgcat}\left(Y\left(a_{i+1}\right)\right) & \leq \operatorname{cgcat}\left(Y\left(a_{i}\right)\right)+\operatorname{cgcat}\left(Y\left(a_{i}, a_{i+1}\right)\right)+1 \\
& \leq(i-1)+0+1=i .
\end{aligned}
$$

Future work. Finally, we mention some future directions suggested by this paper that may be interesting to explore. This paper has focused on the LScategory from a combinatorial viewpoint. Although it is not easy to calculate the standard LS-category of a space, we can use combinatorial techniques to approach the point at which a space is equipped with a combinatorial structure. These discrete methods are suitable for computer calculations such as choosing ideals, finding (weak) beat points and removing them, and repeating these operations. It is advantageous to calculate the LS-category using a computer.

Our strong combinatorial LS-category cCat(-) is a simple homotopy invariant that is analogous to the strong LS-category Cat(-). On the other hand, the simplicial LS-category scat(-) introduced in [7] is a strong homotopy invariant that is analogous to the standard LS-category cat $(-)$. The relation between cCat(-) and scat(-) has not yet been clarified. However, we naturally expect that there will be a similar relation to that between Cat $(-)$ and cat(-) described in Section 2.

In connection with the above, we may need to establish some combinatorial versions of invariants or techniques related to the LS-category of a space such as the cone length, cup length, weak LS-category, and topological complexity.

\section{REFERENCES}

[1] S. Aaronson and N. A. Scoville, Lusternik-Schnirelmann category for simplicial complexes, Illinois J. Math. 57 (2013), no. 3, 743-753. MR 3275736

[2] J. A. Barmak, Algebraic topology of finite topological spaces and applications, Lecture Notes in Mathematics, vol. 2032, Springer, Heidelberg, 2011. MR 3024764

[3] J. A. Barmak and E. G. Minian, Minimal finite models, J. Homotopy Relat. Struct. 2 (2007), no. 1, 127-140. MR 2369155

[4] J. A. Barmak and E. G. Minian, Simple homotopy types and finite spaces, Adv. Math. 218 (2008), no. 1, 87-104. MR 2409409

[5] M. M. Cohen, A course in simple-homotopy theory, Graduate Texts in Mathematics, vol. 10, Springer-Verlag, New York, 1973. MR 0362320

[6] O. Cornea, G. Lupton, J. Oprea and D. Tanré, Lusternik-Schnirelmann category, Mathematical Surveys and Monographs, vol. 103, American Mathematical Society, Providence, RI, 2003. MR 1990857

[7] D. Fernández-Terneroa, E. Macías-Virgósb and J. A. Vilches, Lusternik-Schnirelmann category of simplicial complexes and finite spaces, Topology Appl. 194 (2015), 37-50. MR 3404603

[8] R. Forman, Morse theory for cell complexes, Adv. Math. 134 (1998), no. 1, 90-145. MR 1612391

[9] T. Ganea, Lusternik-Schnirelmann category and strong category, Illinois J. Math. 11 (1967), 417-427. MR 0229240 
[10] L. Lusternik and L. Schnirelmann, Méthodes Topologiques dans les Problémes Variationnels, Hermann, Paris, 1934.

[11] M. C. McCord, Singular homology groups and homotopy groups of finite topological spaces, Duke Math. J. 33 (1966), 465-474. MR 0196744

[12] R. E. Stong, Finite topological spaces, Trans. Amer. Math. Soc. 123 (1966), 325-340. MR 0195042

[13] F. Takens, The Lusternik-Schnirelmann categories of a product space, Compos. Math. 22 (1970), 175-180. MR 0290365

[14] J. H. C. Whitehead, Simple homotopy types, Amer. J. Math. 72 (1950), 1-57. MR 0035437

Kohei Tanaka, Institute of Social Sciences, School of Humanities and Social Sciences, Academic Assembly, Shinshu University, 3-1-1 Asahi, Matsumoto, Nagano 390-8621, JAPAN

E-mail address: tanaka@shinshu-u.ac.jp 